\title{
Pevista
}

ISSN-L: 2215-4728

DOI: http://dx.doi.org/10.15359/rp.22.4

http://www.revistas.una.ac.cr/perspectivas

N. ${ }^{\circ} 22$. Enero-Junio, 2021 • pp. 1-31

\section{La enseñanza de los Estudios Sociales: una aproximación a su interpretación académica}

\author{
The Teaching of Social Studies: An Approach to its \\ Academic Interpretation
}

\author{
Carlos Daniel Solano-Pérez*
}

Fecha de recepción: 15/02/2021 • Fecha de aceptación: 20/03/2021

Resumen: Pensar la enseñanza de los estudios sociales (EES), desde su posición de campo académico es una deuda en los debates nacionales, lo que revela una significativa limitante para alcanzar discusiones de índole teórica y epistemológica. El presente artículo busca aportar al debate de los aspectos investigativos, a través del análisis de un estado actual de la investigación, a partir de los trabajos finales de graduación. En consecuencia, la finalidad de esta investigación recae en la estructuración de una ruta de interpretación epistemológica alternativa, a partir del concepto de didáctica de los estudios sociales.
Abstract: Thinking about the Teaching of Social Studies, from its academic field position, is a debt in the national debates, which reveals a significant limitation to reach discussions of a theoretical and epistemological nature. This article seeks to contribute to the debate of the investigative aspects, through the analysis of a current state of research, based on the Final Graduation Projects. Consequently, the purpose of this research lies in the structuring of an alternative epistemological interpretation route, based on the concept of Didactics of Social Studies.

* Costarricense. Bachiller en la Enseñanza de los Estudios Sociales y Educación Cívica por la Universidad de Costa Rica (UCR), Sede Central «Rodrigo Facio», San José, Costa Rica. Investigador independiente. Correo electrónico: carlos.solanoperez@ucr.ac.cr. ORCID: https://orcid.org/0000-0001-9957-9857. 
Palabras claves: trabajos finales de graduación; epistemología; campo de estudio; investigación; estudios sociales.
Keywords: final degree projects; epistemology; field of study; research area; Social Studies.

«La reflexión epistemológica es un asunto capital para hacer coherente teoría y práctica en la formación del profesor y en su metodología posterior. La universidad tiene ante sí el reto de realizar esa reflexión...» - Pilar Maestro González

\section{Introducción}

Pensar la enseñanza de los estudios sociales (EES), más allá de una asignatura escolar, y situarla como campo académico-universitario, resulta una deuda significativa dentro del marco investigativo nacional. Este comportamiento propicia una nebulosa respecto a la clarificación de su objeto y campo de estudio, lo que aún hoy no se ha resuelto (González, 2020a; López, 2018; Morales, 2010). Incluso, ha llevado a afirmaciones sobre la inexistencia de un campo o saber propio.

De modo significativo, elementos tales como la ausencia de un balance riguroso y sistematizado que genere una fotografía fidedigna de la investigación dentro del espacio de la EES. Y, la escasa o nula discusión teórico-epistémica sobre los fundamentos del campo de la EES imposibilita alcanzar un estadio académico donde los resultados de la investigación permitan la retroalimentación y reelaboración teórica, conceptual, metodológica, así como de sus lógicas investigativas.

En consecuencia, un primer paso es interpretar la EES desde la lógica descrita. Para tal fin, la investigación entiende una disciplina como una construcción socio-histórica, es decir, «un acto de producción cultural» que está en constante movimiento (Apple, 1996). Y, según Ivor Goodson, como saberes-poderes, o sea, «campos de conocimiento observables en el discurso teórico o científico, pero fundamentalmente en la práctica cotidiana y reglamentada» (Velasco, 2014, p. 86). Concretamente, se entiende como disciplina: 
... un campo de conocimiento sistemático que se caracteriza por estudiar determinados objetos de conocimiento, con ciertos métodos y determinadas lógicas de descubrimiento y de justificación, e incluso de aplicación, y con un tipo de discurso que también le es propio. (Camillioni, 2010, p. 59)

Por tanto, en las siguientes líneas se realiza un análisis crítico en torno a las relaciones que se establecen entre el estado actual de la investigación (comprendido por los trabajos finales de graduación (TFG) en la licenciatura de la Universidad Costa Rica (UCR), publicados entre 2000 y 2019) y la construcción de un campo académico y área de investigación en la EES.

Las discusiones desarrolladas son parte de un proyecto de investigación de grado del programa de licenciatura en la UCR. Principalmente, se rescata la contraposición de los resultados del estado actual de la investigación y los debates internacionales sobre la investigación en el campo de la didáctica de las ciencias sociales (DCS) y los social studies, con el afán de poner la experiencia nacional en diálogo con estos espacios de discusión académica internacional.

En cuanto al marco referencial, está en consonancia con los aportes realizados en el campo de la DCS y los social studies. Justamente, se recurre a las discusiones y aportes realizados por autores como Joaquín Prats, Joan Pagés, Alicia Camillioni, Wayne Ross, Stephen Thorthon e Ivo Matozzi. Todo este grupo se vincula a las discusiones de orden epistémico, teórico, metodológico e investigativo. Específicamente, se abordan elementos sobre los propósitos y finalidades de este campo del saber; la interpretación de la didáctica contemporánea; la construcción de un enfoque epistémico propio; y las tendencias y debates de investigación.

Metodológicamente, la investigación identifica las unidades de análisis -59 TFG-, y las sistematiza para construuir la matriz de clasificación y caracterización investigativa de los trabajos finales de graduación en la UCR. A partir de esta matriz, se estructura la tabla Tendencias y debates de investigación en la enseñanza de los estudios sociales, conformada en torno a tres criterios, a saber: núcleo temático, núcleo paradigmático y núcleo praxológico. Posteriormente, se implementa un estado del arte como método de análisis de la tabla, lo que resulta en la 
conformación del estado actual de la investigación, expresado a través de las tendencias, vacíos, alcances, limitantes y retos de investigación.

Finalmente, los resultados de la investigación surgen a partir del análisis y confrontación de tres fuentes de información distintas, estas son: estado actual de la investigación (datos empíricos), el marco referencial (fundamentos teóricos), y las voces y experiencias de algunos especialistas nacionales en el campo de la EES. Conviene señalar que para la construcción de la propuesta de agenda de investigación se construyó la tabla Líneas, temáticas y problemáticas de investigación en la didáctica de los estudios sociales, donde se sistematizaron las tendencias dominantes, las líneas emergentes, los vacíos y retos de la investigación en el campo de la DCS, la EES y los social studies.

Por último, el artículo se conforma de tres apartados. Primeramente, se indagan cuatro aspectos claves, a saber, las aproximaciones conceptuales, los ejes temáticos de investigación, los referentes teórico-metodológicos y el uso del conocimiento construido. Estos elementos permiten evidenciar las relaciones entre el estado de la investigación y la conformación de un campo académico. En un segundo apartado, se elabora un esbozo del escenario actual de interpretación académica que recae sobre la EES en Costa Rica y responde al cuestionamiento «¿Dónde está la EES como campo académico?». Posteriormente, en un tercer apartado se estructura una propuesta en torno al campo de estudio y el área de investigación, según un modelo de interpretación alternativo.

\section{Marco relacional: Entre el estado de la investigación y la confor- mación de un campo académico}

Dentro de la disertación, el punto de partida es la conceptualización misma sobre la EES, pues la determinación de una noción conceptual está relacionada íntimamente con las problemáticas, los métodos y presupuestos teóricos que se adopten, lo cual, en conjunto, delimita un campo de estudio (Quiroz y Díaz, 2011). Ante esto, la discusión debe ir sobre: ¿Qué alcances y limitantes propician las aproximaciones conceptuales sobre la EES para la delimitación de un campo académico?

Antes, y a pesar de ser un campo que mantiene varias décadas de vida, cabe señalar el dominio de concepciones difusas dentro de la EES 
y el poco debate en artículos científicos, seminarios y simposios especializados sobre los principios conceptuales. Como resultado, aún hoy, el proceso de conceptualización de la EES se mantiene en construcción (López, 2018).

$\mathrm{Al}$ respecto, se sigue con una línea conceptual tradicional, que mantiene el statu quo en términos academicistas y funcionalistas. Por tanto, es acertado pensar el proceso de conceptualización desde el conflicto, o sea como:

Un parámetro delimitador de aspiraciones y perspectivas, cuya naturaleza se manifiesta en la medida en la que excluye nociones peligrosas del significando tradicional, e incluye aquellas funcionales en cuanto permitan actualizar el contenido sin romper con la comodidad de la tradición sedimentada. (González, 2020a, p. 11)

Así las cosas, los trabajos finales, lejos de brindar luces de consenso, presentan un alto sentido de ambigüedad en la interpretación que realizan, acercándose a las nociones tradicionalistas.

En primera instancia, algunos trabajos finales evidencian aproximaciones conceptuales explícitas. Lo relevante de esta conceptualización es la posición manifiesta de una postura sobre qué entienden por el campo de la EES. Por un lado, la concepción se construye desde las nomenclaturas de estudios sociales o enseñanza de los estudios sociales, y se vinculan con mayor fuerza a la dimensión de asignatura escolar, es decir, ambos conceptos se refieren a una asignatura básica de los programas de educación estatal vigente. Por ende, delimitada a partir de posturas curriculares del Estado.

De modo que las interpretaciones se conforman a partir de las construcciones ideológicas del MEP o, según el caso, desde la visión de tecnócratas, historiadores y geógrafos. Un rasgo particular de esta conceptualización institucional es concebir un campo de trabajo escolar e interdisciplinar, estructurado por la historia y la geografía, el cual no plantea un objeto de estudio (MEP, 2017).

Por otro lado, a partir de las mismas nomenclaturas conceptuales, algunos trabajos finales evidencian definiciones donde se trasciende la dimensión escolar, aunque sin manifestar con lucidez su condición 
epistémica como campo del conocimiento. Así, se encuentran definiciones de la enseñanza de los estudios sociales como la siguiente:

Una rama del conocimiento específico con objetos y métodos particulares y que su fin no es solo la transmisión simple de conocimientos obtenidos por otras disciplinas, sino que debe de ocuparse de construir conocimientos nuevos, dentro de su singular espacio, entre las Ciencias Sociales y las Ciencias Pedagógicas. (Chinchilla y Martínez, 2011,p. 52)

Justamente, a pesar de la ausencia de clarificación sobre ser un campo de trabajo, una asignatura escolar o una disciplina académica, se visibilizan los intentos de comprender la EES como un saber o campo del conocimiento académico-universitario y centrado en la formación ciudadana a partir de sus disciplinas estructurantes.

Dentro de esta línea interpretativa, se entiende la EES como un campo de trabajo multi e interdisciplinario de las ciencias sociales o como disciplina de las ciencias sociales. Desde esta lógica, se construye un campo de trabajo ampliamente mediado, en su concepción, por los contenidos por enseñar. O sea, un saber fragmentado entre la enseñanza histórica y geográfica, definido según los contenidos curriculares, en consonancia con la tendencia seguida por las didácticas específicas (Davini, 2015).

A pesar de la retórica interdisciplinar que conceptualiza la EES, el estado de la investigación muestra un campo del saber construido sobre la fragmentación disciplinar (historia y geografía). La realidad investigativa da cuenta que tal interdisciplinariedad dentro de las ciencias sociales o humanas, vinculadas a cuestiones de aprendizaje y enseñanza, está lejos de ser una realidad consolidada (Davini, 2015). Muestra de ello es la amplia mayoría de trabajos finales que opta por conceptualizar la noción de enseñanza histórica o geográfica, e inclusive definen historia o geografía, en lugar de discutir sobre la conceptualización de enseñanza de los estudios sociales, de forma integral y holística.

En este punto, conviene señalar el ejercicio investigativo de conceptualizar la EES a partir de marcos interpretativos externos, lo que la investigación denomina como disonancia epistémico-conceptual. En otras palabras, se observa la utilización de una aproximación conceptual 
construida para una disciplina académica, como lo es la DCS (con un objeto y campo de estudio delimitado significativamente y ligado con una realidad socio-histórica particular), para definir un campo de trabajo lo insuficientemente explorado y estudiado con la intención de generar consensos y progresos que permitan ostentar el status de disciplina académica, tal como la EES.

Precisamente, esta disonancia epistémico-conceptual se refiere a la incongruencia de conceptualizar la EES a partir de la aproximación construida para la DCS, cuando la diferencia entre ambos saberes radica en que el segundo trasciende las fronteras epistemológicas del primero. Ante esto, la investigación considera que el campo de la EES tiende a comprenderse sin la necesidad de debates o discusiones rigurosas con respaldo teórico-empírico.

Finalmente, aunque las aproximaciones conceptuales (explícitas e implícitas) no niegan la existencia de un saber académico-universitario, no permiten mayor lucidez sobre la delimitación de este mismo. Los alcances encontrados se limitan a reconocer la existencia de un campo específico del conocimiento que encuentra su posición particular entre las ciencias sociales y las ciencias de la educación, comprendido alrededor de los contenidos por enseñar, a saber, la enseñanza histórica y geográfica. Lo anterior, sin duda, respalda la relación entre la ausencia de clarificación conceptual y la débil delimitación de un campo de estudio (González, 2020a; Morales, 2010).

Seguidamente, otro cuestionamiento se relaciona con los ejes temáticos de investigación explorados y utilizados para construir los trabajos finales. Por consiguiente, el debate se estructura respecto a: ¿Qué significan los ejes de estudio evidenciadas en el estado actual de la investigación para la precisión y delimitación de un campo de investigación?

Efectivamente, se puede afirmar que la lógica investigativa muestra un patrón seguido por los trabajos finales (en su amplia mayoría), donde se problematiza un fenómeno didáctico, luego se presenta una teoría pedagógica (psicología cognitiva) que funciona de sustento conceptual. En seguida, se genera un diagnóstico empírico del tema estudiado, y se finaliza con la construcción de una estrategia o intervención didáctica o educativa que busca validar los propósitos investigativos. Esta secuencia de investigación fue señala por González (2020a), al 
estudiar la lógica investigativa de los TFG, en la Universidad Nacional (UNA).

En efecto, se evidencia una investigación de tipo educativa/didáctica. Pero, la interrogante que se mantiene es: ¿Qué particularidades presenta este tipo de investigación?

$\mathrm{Al}$ respecto, la investigación observa el estudio de la práctica de la enseñanza desde una racionalidad didáctica donde la preocupación es la transmisión de los conocimientos disciplinares construidos desde la historia y la geografía. Es decir, se potencia el estudio de la adecuación de los conocimientos sociales (disciplinares) en la enseñanza escolar, a partir de una lógica didáctica instrumentalizada que, en lugar de buscar la explicación y comprensión de los fenómenos áulicos, se ha concentrado en generar supuestos técnicos (propuestas didáctico-metodológicas) con cierto grado de presunción, en términos de funcionalidad pedagógica, pues no genera resultados comprobables.

Según lo expuesto, se logra evidenciar una investigación didáctica sesgada e insuficiente, pues a la hora de abordar los fenómenos áulicos, no logra ahondar de forma integral los objetos de investigación. Como menciona Merchán (2010), este tipo de estudios no puede estar supeditado a reconocer las labores de docentes y e estudiantes, ni reducir los estudios sobre innovación didáctica a una descripción de roles áulicos, lo que se muestra en el estado de la investigación.

Hasta ahora, se percibe una investigación didáctica desde una perspectiva mecanicista, cuyo propósito es construir saberes técnicos, es decir, centrada en el desarrollo de técnicas pedagógico-didácticas de forma acrítica. Por ende, el marco de referencia es una didáctica mecanicista, es decir, el estatuto didáctico se interpreta como un ejercicio o praxis transpositiva entre un saber sabio y un saber escolar (jerarquización del pensamiento social), dando como resultado un proceso didáctico desvinculado de la realidad áulica, pues se concibe como un acto intuitivo o espontaneo, sin reflexión teórico-epistémico.

Aunque se evidencian esfuerzos que logran trascender esta racionalidad investigativa, es necesario señalar que son una minoría de trabajos. Cuando mucho, se logra advertir que la investigación didáctica expresada desde el campo de la EES presenta un amplio potencial de líneas de investigación que, hasta la fecha, han sido escasamente 
exploradas, aunque los últimos años muestran un incipiente interés por fomentar nuevos abordajes.

La investigación observa que esta perspectiva didáctica mecanicista encuentra su génesis en la lógica normativa impositiva que gobierna el campo y la investigación de la EES. Dicho fenómeno se expresa a través del espacio académico, a raíz de las reticencias académicas ante las didácticas específicas, es decir, posturas didácticas que consideran que para enseñar cualquier tema basta con aplicar un diseño instruccional y con dominar el conocimiento concreto de la disciplina específica (González, 2010).

O bien, se puede interpretar como una especie de subordinación epistémica, donde «el saber histórico se coloca como el nodo central de la enseñanza en la asignatura, instaurando la idea que lo pedagógico es solamente un problema de índole práctico: cómo mediatizar el conocimiento disciplinar y lograr así la formación ciudadana» (González, 2020a, p. 4).

Asimismo, la lógica normativa se expresa desde el espacio estatal, ya que la EES mantiene su génesis en la asignatura escolar, la cual está ligada a prioridades sociales, políticas e ideológicas. Por ende, el estudio exclusivo de los contenidos de enseñanza escolar, sin cuestionar su pertinencia en la formación social y ciudadana, plantea la aceptación del poder regulador del Estado dentro del sistema educativo.

Resulta interesante señalar que los ejes de investigación reflejan los intereses de las lógicas normativas expuestas. Para ilustrar, dentro del estado actual de la investigación predomina el desarrollo de técnicas pedagógicas con fines transpositivos, o sea, sin reflexión teórica o empírica; los conocimientos históricos y geográficos se toman como válidos y no se cuestionan; y el componente didáctico se preocupa por la transmisión de conocimientos construidos en otras disciplinas sociales. Aún más esclarecedora es la ausencia de estudios de índole teórico-epistémico o metodológico.

Otro punto de cuestionamiento surge de los diseños de investigación. ¿Cómo inciden los diseños teórico-metodológicos encontrados dentro del estado actual de la investigación en la conformación de un campo académico y un área de investigación en la EES? 
El estado de la investigación refleja que los objetos de investigación abordados por los trabajos finales cuentan con amplias limitantes en torno a los fundamentos que problematizan el tema de estudio, pues se parte de supuestos retóricos, más no así sustentados en lo teórico. Incluso, se formulan apartados de planteamiento del problema donde solo se expone una pregunta generadora sin ninguna problematización o discusión rigurosa. Además, el estado de la investigación muestra una amplia ausencia, por parte de los trabajos finales, de la explicitación del objeto de estudio, lo cual no tiene razón de ser dentro de una investigación científica.

En sí, la incidencia recae en que la construcción de un objeto de estudio no es arbitraria ni unilateral, debe fundamentarse en una problematización dialéctica e implicar una ruptura con el orden del sentido común (doxa), pues no basta con conjurar la existencia de algún problema, sino hay que demostrarlo teóricamente (Bourdieu et al., 2003).

Otro rasgo evidenciado responde a los marcos de referencia desde donde se teoriza el conocimiento construido. Los datos recabados muestran una tendencia al trasvasamiento de teorías y conceptos, es decir, la adopción de teorías y conceptos que influencian la investigación en distintas disciplinas, debido a la permeabilidad entre las fronteras de campos disciplinares (Camillioni, 2015).

Esta fragmentariedad en los marcos de referencia es producto de la implementación de conceptos y teorías provenientes de las disciplinas estructurantes (historia y geografía) y la amplia rama de las ciencias de la educación (tales como la psicología educativa, sociología de la educación, teoría curricular, entre otras). De modo que se encuentra «un conjunto de teorías de diferentes orígenes y baja articulación» que ocasionan controversia en el terreno epistemológico (Davini, 2015).

Esta amalgama de fundamentos resulta lícita, pues, es válido nutrirse de presupuestos conceptuales y teóricos de otras Ciencias Sociales, en tanto se conserve una lectura de la realidad social propia (Camillioni, 2013). Precisamente, este es el elemento cuestionable, si se ha logrado establecer con claridad una perspectiva social propia. La respuesta puede encontrarse en lo que Aisenberg (1998) denomina como aplicacionismo disciplinar. Ya que, la investigación en la EES toma como base exclusiva los aportes de las disciplinas estructurantes 
para formular los contenidos del área, desconociendo que la investigación didáctica requiere una teoría específica, consciente de la relación didáctica.

Otro factor evidenciado por la investigación es la tendencia por estructurar modelos metodológicos para estudiar la adecuación de los conocimientos sociales en la enseñanza escolar, y la validez de estas investigaciones en términos de aportes al conocimiento construido. Al respeto, algunos especialistas señalan que este comportamiento investigativo no posibilita resultados comprobables, no permite la acumulación de un conocimiento propio en el campo de la EES y deja al campo en el empirismo, dado que este tipo de modelos de investigación imposibilitan la retroalimentación epistémica, teórica y metodológica.

Esta afirmación es comprobable, ya que se logra evidenciar que los diseños de estos trabajos (en su mayoría) se enmarcan en una lógica acrítica, dado que no se cuestiona a profundidad y con rigurosidad metodológica el fenómeno en estudio. De cualquier modo, es acertado cuestionar los diseños de investigación y la producción de conocimiento en términos científicos, dado que no presentan parámetros de aplicabilidad o funcionalidad para su validez, pues los diseños no se proponen una contrastación dialéctica (empírico-teórica) sobre la enseñanza escolar. Por ende, a lo sumo, representan «hipótesis plausibles o interesantes» (Aisenberg, 1998).

En definitiva, la incidencia de estos diseños de investigación es significante, en tanto:

... la calificación de lo científico de un determinado conocimiento no viene dada por la exactitud e inapelabilidad del resultado finalmente conseguido, sino por el camino que se ha trazado para fabricarlo, es decir, por la aplicación de un método: el método científico. (Prats, 2003, p. 2)

Para resumir, los diseños teórico-metodológicos evidencian rasgos de un campo de investigación con un status científico cuestionable. Pues los diseños no permiten obtener resultados comprobables que posibiliten la acumulación y retroalimentación del conocimiento, en tanto refuerzan la interpretación de un campo de investigación donde predomina el estudio de la transmisión de conocimientos sociales desde 
estrategias pedagógicas acríticas. Asimismo, los diseños no alcanzan a desarrollar un marco teórico-conceptual que permita abordar sus objetos de estudio de forma integral (aplicacionismo disciplinar), lo que sostiene la interpretación de una didáctica instrumentalizada.

Finalmente, otro cuestionamiento es entender la lógica del conocimiento construido, dado que las investigaciones y el conocimiento construido demuestran una calidad científica para la consolidación de un campo de estudio (Prats, 2002). En este sentido, cabe reflexionar sobre: ¿Qué utilización le da el campo de la EES al conocimiento construido en sus investigaciones?

Ciertamente, se evidencia que la utilización del conocimiento posee una dimensión educativa/didáctica. No obstante, es necesario profundizar en los rasgos de este conocimiento, con el fin de comprender toda su dimensión y alcances.

Efectivamente, el conocimiento encontrado se puede denominar como un conocimiento educativo.

... el uso que se da a un saber, aplicando sobre él la normativa procedente de las Ciencias de la Educación, no tiene como objetivo la ampliación y profundización de su marco propio, sino la construcción de un sistema específico que permita convertir sus conjuntos informativo en conocimientos formativos dotados, por ello, de significados, en la mente de sujetos perceptores. (González, 2010, p. 12)

Así conceptualizada, esta noción logra englobar la utilización del conocimiento desde la investigación en la EES, dado que la mayoría de aportes encontrados presentan un proceso donde a los contenidos disciplinares se les aplica una teoría de aprendizaje en busca de conformar propuestas de intervención pedagógico-didáctica. Esta utilización del conocimiento evidencia un sentido didáctico/instructivo, pues su preocupación está centrada «en el dominio de técnicas pedagógicas para la transmisión del conocimiento a un sujeto que recepciona» (Pipkin, 2009, p. 38).

Este uso del conocimiento deja entrever una percepción de la EES como un campo encargado de impartir instrucción, una especialidad que «se preocupa por los problemas que surgen ante la transmisión de sus 
contenidos, la transposición de su estructura lógica, y los procesos de comunicación a los que da origen entre emisor y unos sujetos perceptores» (González, 2010, p. 17).

Sin embargo, es de interés señalar dos aspectos.

Por una parte, el conocimiento educativo propuesto por González (2010) cuenta con una lógica epistémica diferente a las ciencias de referencia, dado que su propósito es distinto y, por ende, se crea desde una ciencia para el aula, por tanto, la EES es un saber aplicado, ya que encuentra su razón de ser en los fenómenos áulicos.

Por otra parte, en función de la potencialidad crítica demostrada en algunas líneas de trabajo encontradas en el estado de la investigación, es pertinente señalar la posibilidad de trascender este conocimiento educativo y alcanzar un conocimiento de carácter socio-crítico, entendido como un conocimiento de los fenómenos didácticos con perspectiva social, holística y transformadora.

En este punto, conviene esclarecer el propósito de la EES según el conocimiento construido desde el estado de la investigación. En tal caso, la praxis investigativa evidencia un campo de trabajo centrado en los contenidos disciplinares por enseñar e interesado por el desarrollo de estrategias didácticas. O sea, el propósito es comunicativo-instructivo. En contraposición, desde el discurso, las finalidades se citan como la formación de ciudadanías críticas, participativas y transformadoras, preocupadas por comprender los problemas de la sociedad contemporánea. Es decir, el propósito es crítico-transformador.

\section{La conformación de un campo académico: ¿Dónde nos encontramos?}

A la luz de los hallazgos de la investigación, concretamente se encuentra que según las relaciones estudiadas no se puede plantear la existencia de un campo académico y un área de investigación clarificada y delimitada. Empero, se evidencia un campo de trabajo académico e investigativo que se preocupa por los procesos de enseñanza y aprendizaje de la historia y la geografía en el contexto de la educación obligatoria, y la formación profesional docente. 
Así, se encuentra un campo del conocimiento educativo/didáctico que trabaja académicamente. Este campo de trabajo presenta una postura epistémica particular, o sea, cuenta con una naturaleza distinta (que le es propia), resultado de la aplicación de los fundamentos de las ciencias de la educación a los contenidos de las ciencias de referencia.

Epistemológicamente, el estado de la investigación evidencia que el campo de la EES se ha construido desde un modelo interpretativo con una base explicativa disciplinar-fragmentada. Es decir, se continúa apostando por definir el campo a partir de los componentes y elementos de los saberes que lo integran. De acá, la facilidad de sostener un discurso académico hegemónico de la interpretación «adecuada» sobre la EES. Interpretación y discurso construido por profesionales de historia, geografía y política educativa escolar.

Por consiguiente, al entender la investigación la EES desde una perspectiva académica, conlleva a dos posibles marcos interpretativos. Primeramente, uno enfocado en la existencia de un campo de trabajo a nivel universitario donde convergen los saberes académicos de la educación histórica y geográfica, y preocupado por adecuar los conocimientos disciplinares a la enseñanza, por medio del desarrollo de estrategias pedagógicas. Esta perspectiva se debate entre percepciones que consideran innecesario el desarrollo de un marco teórico propio, pues no es una disciplina académica, y posturas que reflexionan sobre la potencialidad de conformarse en una disciplina académica, con un objeto de estudio y un campo de investigación.

En segunda instancia, se encuentra un marco interpretativo que postula un campo interdisciplinar, que toma sus componentes de las ciencias sociales. Aunque tal sentido se sostiene únicamente en el discurso narrativo, pues, en la práctica sigue siendo enseñanza de la historia y la geografía, aunque con un dominio de la disciplina histórica (Botey, s. f.; López, 2018; Solano, 2006).

$\mathrm{Al}$ respecto, resalta la dificultad de articular aproximaciones conceptuales claras, consensuadas y producto de una investigación teórico-empírica. Aunque, se debe señalar que no resulta necesario estructurar una especie de conceptualización rígida o taxativa (según algunos especialistas). Por el contrario, el debate debe girar sobre el para qué de este campo del saber. En tal sentido, impera mayor consenso, puesto que el 
propósito de este campo se concibe en posibilitar ciudadanías críticas, activas y transformativas.

Por ende, se vislumbra un punto de encuentro al entender el propósito de la EES en la formación del pensamiento social o histórico crítico y promover nuevas ciudadanías que lleven al cambio social (González, 2020b; Ramírez, 2020). Sin embargo, este discurso entra en contradicciones con los propósitos evidenciados desde el estado de la investigación. Efectivamente, la retórica sobre las finalidades sostiene un sentido de criticidad inherente, aunque la praxis investigativa demuestra lo contrario, ya que las investigaciones parten de una dimensión didáctica instrumentalizada y acrítica.

Desde otro punto de vista, la ausencia de progreso de un campo investigativo consolidado en el área de la EES es un rasgo característico. Así lo evidencia el comportamiento monotemático de investigación y la tendencia por implementar diseños de investigación que no generan resultados comprobables, que lleven a una acumulación de conocimiento contrastable, para avanzar a estadios de investigación avanzados.

En este sentido, algunos estudios del tema plantean que la EES ha concretado una etapa de consolidación y se encuentra en una fase de transición, que significa adentrarse en un proceso de transformación, que explora sistemáticamente el para qué de este saber dentro de la sociedad actual, o sea, un período de cuestionamiento de sus lógicas epistémicas.

$\mathrm{Al}$ respecto, durante los últimos años se han visibilizado esfuerzos dentro de los espacios académicos para acercarse a la dimensión académica e investigativa de la EES. Así, las renovaciones curriculares universitarias y la conformación o ligamen a redes investigativas, tales como la Red Centroamericana en Investigación y Docencia de Estudios Sociales y Ciudadanía Crítica (RECIDEC) y Red Iberoamericana en Didáctica de las Ciencias Sociales (RIDCS), forman parte de los intentos actuales por conectar la experiencia nacional a debates internacionales y a un campo de estudio académico (Comisión Compartida, 2020; López, 2018). 


\section{Repensando el campo de la EES: acercamiento a una ruta de inter- pretación académica}

La tesis propuesta por la presente investigación toma de punto de partida la implementación de una nomenclatura conceptual distinta a la tradicional, con el fin de evidenciar la diferenciación de una condición epistémica de carácter académico. Por tal motivo, se propone el concepto de didáctica de los estudios sociales (en adelante DES).

Dicha nomenclatura conceptual responde al entender el punto de articulación de este campo de conocimiento en la didáctica, ya que es necesario desarrollar una teoría didáctica de su accionar específico. No obstante, la resignificación conceptual no basta por sí misma, y debe centrarse en los contenidos de esta renovación conceptual (González, 2020a). Para ello, es requisito indispensable traer la discusión sobre la naturaleza epistémica de este campo académico.

Algunos círculos académicos niegan tal naturaleza epistémica, generando una conceptualización y definición del campo de estudio alrededor de los contenidos por enseñar, lo cual refleja una serie de conflictos de poder entre las comunidades académicas que integran las disciplinas estructurantes de este campo del saber (Davini, 2015). Empero, esta desnaturalización epistémica no es de extrañar, pues dicho razonamiento encuentra su fundamento en la predominancia de las lógicas normativas impositivas que han regulado la evolución histórica de este saber.

No obstante, a consideración de la presente investigación, la influencia de esta lógica normativa no niega la existencia de una epistemología propia, sino que ha considerado innecesaria la exploración, estudio y debate de dicha naturaleza epistémica. Máxime, al evidenciar la construcción de un conocimiento específico en la DES, por cuanto se considera que es dueña de una epistemología particular (Aisenberg, 1998; González, 2010; Santisteban y Pagés, 2018).

Siguiendo esta línea de ideas, se construye un marco interpretativo alterno a las explicaciones epistémicas sustentadas en la parcelación disciplinar, de poca articulación. Esta apunta por el desarrollo de un marco interpretativo integral y holístico. La figura 1 muestra la 
lógica estructural del modelo interpretativo con una base explicativa perspectiva-social.

\section{Figura 1}

Modelo interpretativo de la DES con base explicativa en una perspectiva social propia

\section{Lógica estructural del modelo}

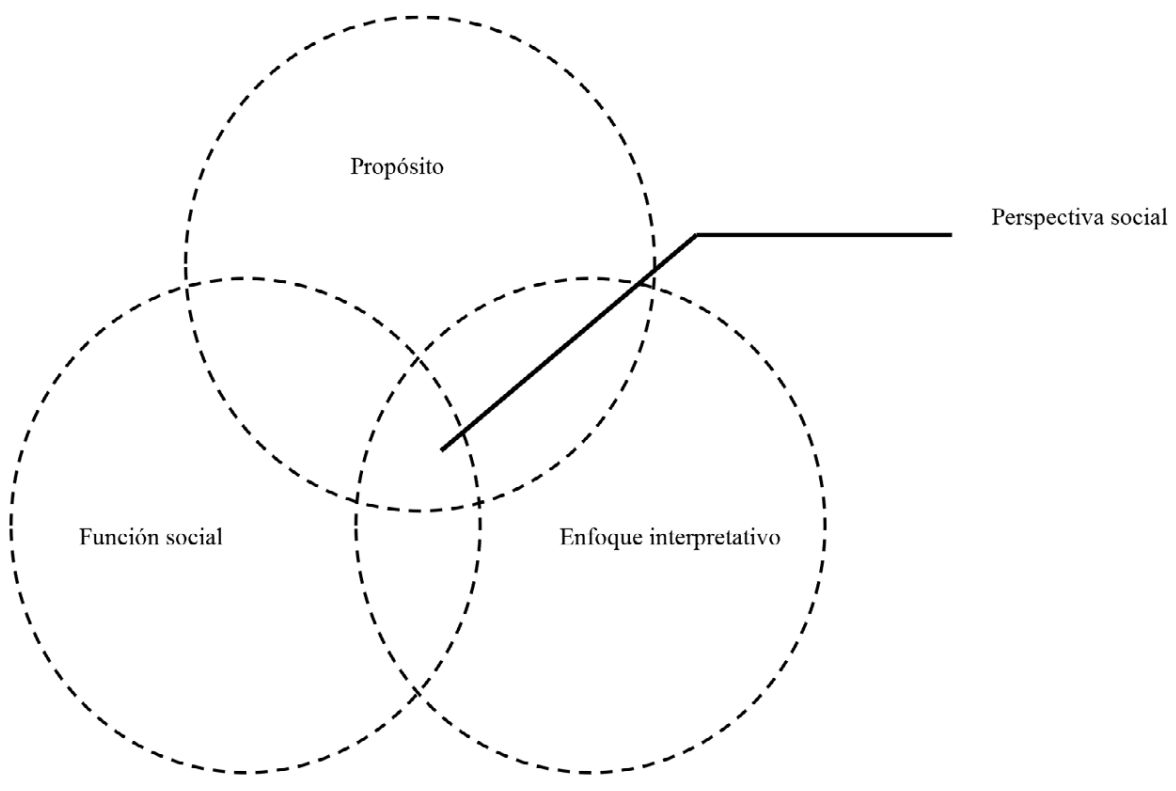

Fuente: elaboración propia.

De esta forma, el modelo propuesto no busca delimitar un objeto de estudio disciplinar tradicional, sino construir una base epistémica a partir de una perspectiva de observación de la realidad social. Ante esto, la investigación interpreta que superar la lógica normativa impositiva pasa por construir un camino epistemológico distinto a los modelos de fragmentación disciplinar, dado que este modelo interpretativo se nutre de los aportes (teóricos, epistémicos o metodológicos) de las ciencias 
sociales, aunque leídos desde una perspectiva social propia, en tanto, construye sus límites teórico-epistémicos, más allá de las disciplinas de las cuales toma aportaciones.

En consecuencia, se aspira construir un campo específico y diferenciado de investigación que obtenga resultados de igual naturaleza, o bien validez, que el resto de las ciencias sociales. (Rodríguez, 2015). Esta condición epistémica pasa por comprender que lo social es una unidad de estudio, y los enfoques o perspectivas de observación de la realidad social determinan los campos, o nuevos campos, de estudiarla (Prats, 2002). Por tanto, el modelo interpretativo plantea un giro epistémico, puesto que se propone comprender, explicar y definir el campo del conocimiento a través de una perspectiva propia de la realidad social y no de los contenidos por enseñar.

Este giro epistémico toma relevancia cuando los contenidos curriculares escolares han sido una suerte de «frontera disciplinar» que no ha logrado clarificar los limites teórico-epistémicos del campo de estudio. Principalmente, debido a que la selección de contenidos curriculares surge de posturas seudocientíficas y, aún las presuntamente objetivas resguardan un tipo de etnocentrismo de historiadores y geógrafos (Camillioni, 2013).

Alcanzar esta perspectiva social propia recae en pensar los propósitos, la función social y el enfoque interpretativo. Así las cosas, de los posibles enfoques en torno a los propósitos de la DES (Pagés, 2009) se toma de punto de partida el énfasis por la formación de un pensamiento social crítico o reflexivo (Pipkin, 2009). Es decir, el propósito es promover ciudadanías críticas de los puntos de vista aceptados por la sociedad dominante, que posibiliten la transformación social democrática (Ross, 2006a). Siguiendo los planteamientos de Wayne Ross, «the purpose of social studies is citizenship education aimed at providing students opportunities for an examination critique, and revision of past traditions, existing social practices, and modes of problem solving» (Ross, 2004, p. 250).

En consecuencia, el propósito de la DES se enmarca en propiciar condiciones ideológicas y materiales inherentes para educar a la ciudadanía desde la alfabetización crítica, o sea, una educación para la ciudadanía asociada a interpretar el mundo de forma transformativa (Pipkin, 2009). 
Respecto a la función social, se ubica en promover el cambio de la sociedad, en el marco de la justicia social, a través de nuevas ciudadanías cuestionadoras de las desigualdades e injusticias, mediante el abordaje de los problemas socialmente relevantes (Ross, 2006b; Santisteban, 2019). Por tanto, se perfila una función desmitificadora y cuestionadora de «situaciones que, aunque parezcan normalizadas, son nuevas formas de control y de reproducción de las relaciones de poder» (Santisteban y Pagès, 2018).

En suma, plantear con lucidez la intercepción entre el propósito y la función social de la DES conlleva adherirse a los postulados de Pagés, al mencionar que la finalidad de esta área del saber «ha de ser contrasocializadora, es decir ha de preparar al alumno para que construya sus propios conocimientos, se ubique en su mundo y esté preparado para intervenir en él de manera democrática» (2002, p. 258).

Así entendidas las finalidades, pueden enmarcarse dentro de una visión de educación social, puesto que propiciar ciudadanías críticas conlleva conciencia sobre las demandas sociales, en tanto el cambio social necesita actuar en torno a estas mismas. Esta interpretación, con tradición en los planteamientos de Dewey, encuentra la significancia de este campo en la formación del pensamiento reflexivo y su capacidad de transformación social, a través de la identificación de las demandas y problemas sociales, y la preparación para la vida en sociedad (Thornton, 2008).

A pesar de que la enseñanza de lo social se relaciona con los problemas y demandas sociales, no rechaza los aportes de las disciplinas sociales, sino intenta trascender las fronteras disciplinares mediante el estudio de problemas socialmente relevantes que potencien los aprendizajes (Pagés, 2009). En este contexto, se considera que la formación socio-cultural necesita un enfoque que marque el norte, es decir, que articule e interprete la realidad social y cultural, que pueda sistematizarla y problematizarla con un sentido teórico-epistémico determinado.

En este caso, la perspectiva para abordar los problemas socialmente relevantes se posiciona desde los postulados del enfoque geohistórico, como caleidoscopio del pensamiento social, pues a partir de esta perspectiva se logra comprender el desarrollo de lo social y ecológico a lo largo del tiempo y del espacio (Matozzi, 2014). 
Este énfasis permite acercarse a una especie de enfoque propio en la formación del pensamiento social, pues une lo social al espacio y al tiempo, ya que «es el estudio de un doble vínculo, de la naturaleza con el hombre y del hombre con la naturaleza, el estudio de una acción y de una reacción, mezcladas, confundidas, incesantemente reanudadas, en la realidad de cada día». (Braudel, citado en Matozzi, 2014, p. 13).

En palabras de Matozzi (2014), la significancia de la geohistoria recae en:

La integración del pensamiento geográfico con el pensamiento histórico [que] puede generar: conocimientos de historia profunda; conocimientos significativos y útiles para comprender el mundo; la importancia de las descripciones y descripciones densas; la importancia de los factores geográficos en la explicación histórica; la relevancia de las dinámicas históricas en la explicación de las estructuras territoriales, de los paisajes y de los entornos; la emoción ante la comprensión más profunda y ante el aprendizaje de conocimientos geográficos e históricos. (p. 102)

Según lo expuesto, se comienza a dibujar un campo del conocimiento basado en una formación social y cultural, que mantiene su propósito en la formación ciudadana participativa y transformadora, y en el pensamiento social crítico, desde una perspectiva geohistórica, que se plantea la desmitificación y cuestionamiento de las estructuras sociales, políticas y culturales que gobiernan las relaciones de poder.

Entonces: ¿Cuál es la perspectiva de observación de la realidad social de la DES? Para el caso, el enfoque particular responde a la formación social y cultural en perspectiva geohistórica en el marco de la educación obligatoria. Desde esta postura, se deja de lado la tendencia de reconceptualizar las didácticas específicas alrededor de los contenidos de enseñanza, lo que en sí mismo genera amplios problemas (Davini, 2015). Ahora, la prioridad epistemológica se basa en debatir y comprender la DES a través de una perspectiva particular de la realidad social.

Así las cosas, entender, reflexionar y postular la DES a partir de su propósito y función social conlleva posicionar este saber en consonancia con un proyecto socio-educativo emancipador. Ante esto, la 
construcción de un conocimiento que trascienda los límites instrumentales del conocimiento educativo encontrado en el estado de la investigación, y propuesto por González (2010), resulta imperante.

En consecuencia, se propone la necesidad de construir un conocimiento socio-educativo, capaz de desarrollar una forma de pensar integrada, la cual interrogue las interrelaciones áulicas y su entorno, o sea, aborda los procesos y fenómenos didácticos atendiendo los nexos con el micro y macro cosmos educativo. Además, cuestiona la significancia socio-cultural de los conocimientos que construye, cómo son mediados por un marco político-ideológico, y las relaciones de poder y legitimación en torno a la formación del pensamiento social. En tanto, es resultado de un proceso epistémico dialectico (Santisteban y Pagés, 2018).

En otro orden de cosas, la tesis propuesta sostiene que la perspectiva propia es la enseñanza y aprendizaje del pensamiento social crítico (formación social). En consecuencia, la didáctica se perfila como eje articulador de la DES. Ello resulta epistemológicamente válido, en tanto la didáctica se configura como ámbito de acción y observación de la realidad que permite construir conocimiento científico de lo social (Prats, 2002).

Empero, es ineludible abstraerse de lo controvertido que resulta, en la actualidad, la definición del status epistemológico de la didáctica, lo que conlleva a clarificar la postura interpretativa con mayor lucidez. Siendo este el caso, se considera pertinente alejarse de los marcos de interpretación positivista o neopositivista, que perciben este campo como una teoría práctica, no científica. Por tanto, se comparte la lógica de la didáctica como una ciencia social, capaz de construir un conocimiento, igualmente, social. A través de una propuesta dialéctica teórico y áulica (Camillioni, 2013; Maestro, 1993).

La postura didáctica propuesta se perfila como una teoría de la enseñanza y el aprendizaje preocupada por los fenómenos de la enseñanza, las condiciones de transmisión de la cultura y las condiciones de la adquisición de un conocimiento por un aprendiz (Camillioni, 2015). Se parte de una racionalidad didáctica que permita pasar de preocuparse por el dominio de técnicas pedagógicas, y alcanzar una perspectiva transformativa, que se preocupe por investigaciones, procesos de formación y condiciones ideológicas necesarias para la formación del pensamiento social crítico y las «nuevas ciudadanías» (Pipkin, 2009). 
En esta línea de ideas, no se comparte la interpretación de que el proceso de aprendizaje se convirtió en un obstáculo para la formulación de una teoría didáctica (Camillioni, 2015). Contrariamente, se considera disonante la comprensión didáctica como espacio preocupado por la enseñanza únicamente, excluyendo los fenómenos anexos al proceso de aprendizaje. De esta forma, la lógica didáctica postulada cuestiona la enseñanza y el aprendizaje, en busca de resultados que permitan actuar sobre ambos procesos educativos, desde una relación teórico-práctica (Aguilera y González, 2008; Aisenberg, 1998).

Así las cosas, a partir de los elementos que dotan de sentido, el constructo conceptual propuesto (giro epistemológico, desarrollo de una perspectiva social y reposicionamiento del componente didáctico) se puede aspirar a alcanzar un carácter autónomo como disciplina social (Pipkin, 2009), en tanto supone culminar con la lógica normativa impositiva.

Según lo expuesto, se entiende la DES como un campo de conocimiento ocupado de teorizar la didáctica de los estudios sociales, por cuanto se preocupa por estudiar, comprender, explicar y cuestionar lo que ocurre en torno a las prácticas de la enseñanza, las experiencias de aprendizaje y las condiciones político-ideológicas de la formación social con un enfoque geohistórico. Además, implica tomar como finalidad la formación del pensamiento social crítico y creativo, el desarrollo de capacidades para interpretar los problemas sociales y tomar decisiones respecto a posibles soluciones (González y Santisteban, 2014).

En términos epistémicos, esta propuesta es pensada desde los postulados de la teoría crítica de la educación o la didáctica crítico-comunicativa, donde el énfasis recae en el desarrollo del pensamiento reflexivo y la formación de una ciudadanía crítica, que permita un cambio social direccionado a la justicia y a la equidad (González, 2013). Dentro de esta línea, se perfila como un saber académico de síntesis, que plantea una relación dialéctica en la construcción del conocimiento, y toma como método la praxis social, por esto, su carácter interventor.

En tanto se toman aportes teórico-epistémicos y metodológicos de otras disciplinas sociales y educativas, estos son leídos desde su perspectiva social particular, por lo que la construcción de su conocimiento plantea una naturaleza epistemológica propia (Camillioni, 2013). 
En suma, acercarse a la definición de la DES es posible, al comprenderla como un campo del saber académico que estudia los procesos didácticos de la formación social y cultural en perspectiva geohistórica, y en la formación docente en esta área del conocimiento. En tanto, encuentra su finalidad en la formación del pensamiento social reflexivo, la conformación de ciudadanías críticas y transformativas, y el cambio social en busca de la justicia y la democratización de la vida social.

\section{Aproximación a una posible agenda de investigación}

La presente propuesta se articula a través de una serie de núcleos de investigación producto de la reflexión y discusión de las tendencias investigativas dominantes. Además, es resultado de la sistematización de los hallazgos del estado actual de la investigación y las discusiones de las experiencias internacionales. No obstante, esta propuesta no representa directrices a futuro ni se plantea de forma taxativa.

A continuación, y de forma esquemática, se exponen los núcleos de investigación propuestos.

\section{La práctica de la enseñanza y los procesos curriculares}

Este eje de investigación se enfoca en investigaciones sobre las innovaciones y estrategias didácticas para la formación social, el desarrollo de competencias y habilidades para fomentar el pensamiento social crítico, la conciencia histórica y la conciencia geográfica. Además, en investigaciones sobre la construcción de conceptos, procesos y fenómenos como parte de los contenidos de enseñanza, las estrategias y procesos de evaluación, y el análisis y propuesta de modelos curriculares.

La relevancia de este núcleo problemático se fundamenta en la posibilidad de indagar a profundidad aspectos didácticos (procesos, patrones, materiales y recursos) de forma crítica, tales como el uso del libro de texto u otros materiales implementados en el quehacer áulico. Además, de indagar elementos curriculares como la educación ambiental, educación ciudadana, educación social, educación patrimonial, educación histórica, educación geográfica, entre otros. O bien, los referentes alternativos de articulación curricular, por ejemplo, los problemas socialmente relevantes o cuestiones socialmente vivas. 


\section{Experiencias y progresión de los aprendizajes}

El segundo eje de investigación plantea investigaciones sobre los distintos procesos de aprendizaje en el área específica de la DES; sobre los conceptos, procesos y fenómenos relacionados a la formación social, política y cultural. A la vez, discute sobre investigaciones que analizan los diversos modelos, paradigmas y enfoques de aprendizaje; se vincula con la psicología educativa y las neurociencias. De forma similar, estudia las interrelaciones dentro de los procesos de aprendizaje y los elementos que inciden en los sujetos que aprenden.

La pertinencia de este núcleo de investigación recae en abordar los procesos cognitivos y la progresión de los aprendizajes en los distintos espacios y niveles en los que se imparte la DES. Así como en la aplicación de tendencias y paradigmas psicoeducativos a los procesos de enseñanza y aprendizaje de la formación social, la educación histórica y la educación geográfica.

\section{Investigación, teoría y metodología}

Dentro del tercer eje, las investigaciones realizadas se enfocan en el campo de estudio mismo, sus postulados teóricos, epistémicos, conceptuales y metodológicos. En tanto, se investiga sobre la construcción de conocimiento escolar y académico, la acumulación de este mismo y las tendencias de la investigación dentro de este campo.

Igualmente, la pertinencia de estas investigaciones radica en discutir la razón de este campo de estudio (propósitos, función social y finalidades); la configuración del campo de estudio en torno al conocimiento disciplinar o las problemáticas sociales, y los alcances epistemológicos de la investigación social y didáctica; las posibles metodologías de investigación, y revisiones investigativas de líneas de estudio.

\section{Formación docente y prácticas pedagógicas}

Este eje de investigación estudia la formación del profesorado dentro de los distintos espacios de la educación inicial y permanente. A la vez, estudia los diversos aspectos de la profesionalización, tales como lo administrativo, institucional, social, económico, político, educativo, 
entre otros. Además, realiza investigación sobre las prácticas pedagógicas de docentes, sus conductas dentro del aula, los elementos que inciden en su ejercicio -creencias, conocimientos y representaciones-, y el papel docente dentro de las dinámicas áulicas.

Asimismo, la relevancia de estudiar estos componentes es profundizar en aspectos relacionados al desarrollo de competencias como el pensamiento histórico y geográfico, los modelos y estrategias curriculares de formación universitaria y las tendencias educativas contemporáneas en relación con las prácticas pedagógicas reflexivas, entre otras.

\section{Alfabetización tecnológica y desarrollo de entornos virtuales}

Este eje de investigación plantea estudios sobre la aplicación de las nuevas tecnologías en los procesos de enseñanza y aprendizaje, y el desarrollo de las competencias necesarias para la alfabetización digital. Además, se trabaja el diseño y gestión de ambientes virtuales para el aprendizaje de contenidos geohistóricos y el desarrollo de estrategias digitales para la formación social y cultural.

La relevancia de este núcleo de investigación recae en el desarrollo de las competencias informacionales vinculadas al pensamiento social reflexivo, así como al campo de la educación.

\section{Significancia de la formación social y el proyecto político-cultural pedagógico}

Dentro de este eje, las investigaciones se interesan por los proyectos socio-pedagógicos y su incidencia en los modelos de organización escolar. También, se generan investigaciones sobre los intereses político-ideológicos y el efecto de estos factores en la formación social. En este sentido, se generan críticas sobre los contenidos disciplinares de la enseñanza y los programas de estudio, y los procesos áulicos como espacios interventores de la realidad social.

La pertinencia de estas investigaciones sobresale por el sentido contra socializador. Por tanto, algunos de los estudios pueden mantener relación con otras dimensiones investigativas. No obstante, permite entender la relevancia simbólica de la formación social y de cómo ha de estructurarse, y de las investigaciones sobre cuestiones socialmente relevantes. 


\section{Reflexiones finales}

Posterior al análisis de las relaciones establecidas, todos los aspectos (aproximación conceptual, ejes de investigación, diseños teórico-metodológicos y conocimiento construido) apuntan a la posibilidad de pensar la EES desde su dimensión académica, con su eje articulador en la didáctica. En este sentido, se observa un área de investigación enfocada en la construcción de trabajos que diagnostican (descriptivamente) la enseñanza de un contenido disciplinar y formulan una estrategia didáctico-metodológica, sustentada en la psicología cognitiva

Como se observa, la racionalidad didáctica mecanicista responde a preocupaciones por la transmisión acrítica de conocimientos disciplinares a través del desarrollo de estrategias pedagógico-didácticas, y a la jerarquización del pensamiento social, basado en perspectivas academicistas. Esta lógica investigativa didáctico-instructiva se contrapone con la racionalidad identitaria otorgada al campo de la EES, donde se potencia, desde el discurso, un área naturalmente crítica y transformativa.

Esta contradicción entre praxis investigativa y discurso teórico es producto de un desarrollo académico dominado por lógicas normativas impositivas. Dicho proceso de institucionalización académica generó la conformación de un campo del saber con un vacío epistémico (López, 2018), pues la EES se debate entre unas políticas curriculares de Estado, y el choque de poder de las disciplinas estructurantes (historia y geografía) y ha dejado de lado el desarrollo de una perspectiva social propia.

Lo anterior ha postergado el debate sobre los aspectos teórico-epistémicos y, por ende, las discusiones sobre la EES como un espacio académico. Así, la desnaturalización epistémica es resultado de un menoscabo de la potencialidad del campo de la EES. Así, la disputa responde a las finalidades mismas de la EES, o sea, el debate entre un campo del conocimiento para la transmisión cultural o para el desarrollo del pensamiento crítico o reflexivo (Ross, 2006a).

Asimismo, la falta de problematización científica (dialéctica), el aplicacionismo disciplinar y la generación de resultados no comprobables llevan el campo a una especie de empirismo, que limita la trascendencia hacia estadios investigativos que permitan la consolidación de un área de investigación consensuada. 
En síntesis, la EES carece de un campo académico-científico lo suficientemente discutido, consensuado e investigado en Costa Rica. Sin embargo, como se evidencia, se encuentra certeza sobre un área de trabajo/conocimiento con un potencial ineludible para conformarse en una disciplina académica. Por ende, surgen algunas cuestiones al respecto, tales como: ¿Qué características teórico-epistémicas le son propias al campo de la EES? ¿Es requisito consolidar la EES como un saber académico? ¿Qué pertinencia tiene una reconceptualización del campo de la EES en la sociedad actual? ¿Cuáles son las finalidades de la EES dentro del contexto social actual? ¿Cuáles son las posibles rutas para alcanzar un status epistémico y teórico propio? ¿Cuáles son las principales limitantes para la conformación de la EES en un saber académico? ¿Cómo solventar estas falencias?

Finalmente, la relevancia de la propuesta interpretativa postulada radica en entender las interrelaciones entre los propósitos y finalidades, y las lógicas que gobiernan y delimitan el campo de estudio (Pagés, 2019). Sin embargo, es necesario advertir que la búsqueda por repensar el campo en términos epistémico-conceptuales no es suficiente, en ninguna forma, para lograr darle un viraje distinto a este campo del saber.

Por ende, no se cree posible resolver las limitantes destacadas a lo largo de la investigación, en torno a la conformación de un campo académico-científico con la postulación de un concepto renovado. A lo sumo, se aspira a plantear nuevas posturas interpretativas, generar discusiones e inducir reacciones cuestionadoras sobre el futuro de este campo del conocimiento, pues alcanzar estadios investigativos y un status académico consolidado requiere de esfuerzos compartidos entre los diversos actores socioeducativos, vinculados al desarrollo de redes de investigación; mayor producción investigativa de calidad; reestructuraciones en la formación inicial docente y continua, y apertura del marco institucional, expresado desde intercambios internacionales y nuevas ofertas institucionales de posgrado. 


\section{Referencias}

Aisenberg, B. (1998). Didáctica de las ciencias sociales: ¿Desde qué teorías estudiamos la enseñanza? Revista de Teoría y didáctica de las Ciencias Sociales, (3), 136-163.

Aguilera, A. y González, M. I. (2008). Didáctica de las ciencias sociales y/o didáctica como ciencia social. Pedagogía y Saberes, (28), 107-114. https://doi.org/10.17227/01212494.28pys107.114

Botey, A. M. (1997). Algunas reflexiones en torno a la enseñanza de la historia en las escuelas y colegios. Reflexiones, 61(1), 1-11. https://revistas.ucr.ac.cr/index. $\mathrm{php} /$ reflexiones/article/view/10978/10359.

Bourdieu, P., Chamboredon, J. C., y Passeron, J. C. (2003). El oficio del sociólogo (24 ed.). Siglo XXI editores.

Camillioni, A. (2015). De herencias, deudas y legados. Una introducción a las corrientes actuales de la didáctica. En A. Camillioni et al. (Eds.), Corrientes didácticas contemporáneas (pp. 17-39). Paidós.

Camillioni, A. (2013). Epistemología de la didáctica de las ciencias sociales. En B. Aisenberg y S. Alderoqui (Coord.), Didáctica de las ciencias sociales. Aportes y reflexiones. (pp. 25-41). Paidós.

Camilloni, A. (2010). La didáctica de las ciencias sociales: ¿Disciplinas o áreas? Revista de Educación, 1(1), 55-76. https://fh.mdp.edu.ar/revistas/index. php/r_educ/article/view/6/50

Chinchilla, E. y Martínez, W. (2011). Análisis del abordaje de la década de 1940 en Costa Rica, a partir del libro de texto, para la enseñanza de los estudios sociales en educación diversificada y la mediación pedagógica del docente [Tesis de licenciatura]. Universidad de Costa Rica, San José, Costa Rica.

Comisión Compartida. (2020). Bachillerato y licenciatura en enseñanza de los estudios sociales. Propuesta de modificación parcial del currículo. Universidad de Costa Rica. [Documento inédito].

Davini, M. C. (2015). Conflictos en la evolución de la didáctica. La demarcación entre la didáctica general y las didácticas especiales. En A. Camillioni et al. (Eds.), Corrientes didácticas contemporáneas (pp.41-73). Paidós.

González Sánchez, D. (2020a). Los estudios sociales de cara a la historia: Provocaciones para un debate urgente. Revista Actualidades Investigativas en Educación, 20(1), 1-18. https://doi.org/10.15517/aie.v20i1.40176. 
González Sánchez, D. (2020b). La enseñanza de los estudios sociales en la era de la calidad educativa. Revista Perspectivas, 20(1), 1-27. https://doi.org/10.15359/rp.20.2

González, I. (2010). Prospectiva de las didácticas específicas, una rama de las ciencias sociales de la educación para la eficacia en el aula. Perspectiva Educacional, Formación de Profesores, 49(1), 1-31. http://www.perspectivaeducacional. $\mathrm{cl} /$ index.php/peducacional/article/view/2.

González, G. y Santisteban, A. (2014). Una mirada a la investigación en didáctica de las ciencias sociales. Revista Latinoamericana de Estudios Educativos, 10(1), 7-17. https://www.redalyc.org/articulo.oa?id=1341/134139791001.

González, G. (2013). El profesorado en formación y las finalidades de la enseñanza de las ciencias sociales. Uni-pluriversidad, 38(13), 24-34. https://revistas.udea. edu.co/index.php/unip/article/view/16971.

López, M. (2018). La enseñanza de la historia en Costa Rica: Un acercamiento desde los planes de la carrera de Estudios Sociales en la Universidad Nacional (1979-2005). Revista Perspectivas, (16), 1-22. https://doi.org/10.15359/ rp.16.2.

Maestro, P. (1993). Epistemología histórica y enseñanza. En P. Ruiz (Ed.), La historiografía (pp.143-181). Marcial Ponds.

Matozzi, I. (2014). ¿Quién tiene miedo de la geohistoria? Enseñanza de las Ciencias Sociales, (13), 85-105. https://www.redalyc.org/articulo.oa?id=3241/324143972009

Merchán, F. (2010). La práctica de la enseñanza de las ciencias sociales como objeto de investigación de la didáctica: Importancia y dificultades metodológicas. Bases para una teoría de la acción en el aula. En R. Ávila, P. Rivero y P. Domínguez (Coords.), Metodología de investigación en didáctica de las ciencias sociales (pp. 104-113).

Ministerio de Educación Pública. (2016). Programas de estudio de Estudios Sociales tercer ciclo de la educación general básica y educación diversificada. San José, Costa Rica: Imprenta Nacional. https://www.mep.go.cr/programa-estudio/estudios-sociales-1.

Miralles, P., Molina, S. y Ortuño, J. (2011). La investigación en didáctica de las ciencias sociales. Educatio Siglo XXI, 29(1), 149-174. https://revistas.um.es/educatio/article/view/119921/112891.

Morales, L. C. (2010). Apuntes sobre epistemología e investigación en la enseñanza de los estudios sociales. Revista Educación, 34(2), 61-74. http://dx.doi. org/10.15517/revedu.v34i2.487. 
Pagés, J. (2019). Ciudadanía global y enseñanza de las ciencias sociales: Retos y posibilidades para el futuro. Revista de Investigación en Didáctica de las Ciencias Sociales, (5), 5-22. https://doi.org/10.17398/2531-0968.05.5

Pagés, J. (2009). Enseñar y aprender ciencias sociales en el siglo XXI: Reflexiones casi al final de una década. Investigación en Educación, Pedagogía y Formación Docente, II Congreso Internacional. Universidad de Antioquia (pp. 140-154).

Pagés, J. (2002). Aprender a enseñar historia y ciencias sociales: El currículo y la didáctica de las ciencias sociales. Pensamiento Educativo, (30), 255-269.

Pipkin, D. (2009). Pensar lo social. Buenos Aires, Argentina: Universidad Nacional de General Sarmiento..

Prats, J. (2003). Líneas de investigación en didáctica de las ciencias sociales. História \& Ensino. Revista do Laboratorio de Ensino de História, (9), 1-25. http://dx.doi.org/10.5433/2238-3018.2003v9n0p133.

Prats, J. (2002) Hacia una definición de la investigación en la didáctica de las ciencias sociales. Enseñanza de las Ciencias Sociales, (1), 81-89. https://www.raco. cat/index.php/EnsenanzaCS/article/view/126132/183031

Prats, J. (1996). La investigación en didáctica de las ciencias sociales. Notas para un debate deseable. VII Symposium de Didáctica de las Ciencias Sociales la investigación, Centro Superior de Formación del Profesorado, Las Palmas de Gran Canaria, 7-28.

Quiroz, R. E. y Díaz, A. E. (2011). La investigación en el campo de la didáctica de las ciencias sociales y su dinámica de articulación en un grupo de universidades públicas en Colombia. Uni-pluri/versidad, 11(2), 1-16. http://bibliotecadigital.udea.edu.co/dspace/bitstream/10495/2943/1/Quiroz_ Ruth_2011_investigacion_campo_didactica.pdf.

Ramírez, J. (2020). La formación inicial y las prácticas docentes como categoría de investigación en los estudios sociales. Revista Perspectivas, 21(1), 1-24. http://dx.doi.org/10.15359/rp.21.4.

Rodríguez, R. (2015). ¿Es posible un ámbito de investigación en didáctica de la historia? Revista de Estudios Sociales, (52), 209-219. https://doi.org/10.7440/res52.2015.15

Ross, E. W. (2006a). The struggle for the social studies curriculum. En E. W. Ross (Ed.), The Social Studies Curriculum. Purposes, Problems and Possibilites (pp. 17-36).

Ross, E. W. (2006b). Remaking the Social Studies Curriculum. En E. W. Ross (Ed.), The Social Studies Curriculum. Purposes, Problems and Possibilites (pp. 319-332). 
Ross, E. W. (2004). Negotiating the politics of citizenship education. PS: Political Science and Politics, 37(2), 249-251. https://doi.org/10.1017/S1049096504004172.

Salas, J. A. (2000). La incorporación de los estudios sociales como materia de estudio escolar en Costa Rica y la necesidad de su reconceptualización de cara a los desafíos para la educación a inicios del siglo XXI, Revista Perspectivas. Estudios Sociales y Educación Cívica, 3(1-2). https://www.revistas.una.ac.cr/index.php/perspectivas/article/view/3849.

Santisteban Fernández, A. (2019). La enseñanza de las ciencias sociales a partir de problemas sociales o temas controvertidos: Estado de la cuestión y resultados de una investigación. El futuro del pasado, (10), 57-79. https://dialnet.unirioja.es/servlet/articulo?codigo=7105038.

Santisteban Fernández, A. y Pagés, J. (2018). La didáctica de las ciencias sociales y de sus disciplinas. Reflexiones al hilo de la obra de Camillioni. Revista de Educación, 14(2), 45-65.

Solano, E. (2006). ¿Enseñamos estudios sociales o historia? Actualidades Educativas en Educación, 6(2), 1-21. http://dx.doi.org/10.15517/aie.v6i2.9213.

Thornton, S. (2008). Continuity and change in social studies curriculum. En L. Levstik, \& C. Tyson (Eds.), Handbook of research in social studies education (pp. 15-32). 